Florida International University FIU Digital Commons

$11-8-2013$

\title{
The Impact of Maternal Acculturation, Youth Age, Sex and Anxiety Sensitivity on Anxiety Symptoms in Hispanic Youth
}

Maria Pienkowski

Florida International University

DOI: $10.25148 /$ etd.FI13121601

Follow this and additional works at: https://digitalcommons.fiu.edu/etd

Part of the Psychology Commons

\section{Recommended Citation}

Pienkowski, Maria, "The Impact of Maternal Acculturation, Youth Age, Sex and Anxiety Sensitivity on Anxiety Symptoms in Hispanic Youth" (2013). FIU Electronic Theses and Dissertations. 1040.

https://digitalcommons.fiu.edu/etd/1040 


\section{FLORIDA INTERNATIONAL UNIVERSITY}

Miami, FL

THE IMPACT OF MATERNAL ACCULTURATION, YOUTH AGE, SEX AND ANXIETY SENSITIVITY ON ANXIETY SYMPTOMS IN HISPANIC YOUTH

A dissertation in partial fulfillment of the requirements for the degree of DOCTOR OF PHILOSOPHY

in

PSYCHOLOGY

by

Maria Pienkowski

2013 


\section{To: Dean Kenneth Furton}

College of Arts and Sciences

This dissertation, written by Maria Pienkowski, and entitled The Impact of Maternal Acculturation, Youth Age, Sex and Anxiety Sensitivity on Anxiety Symptoms in Hispanic Youth, having been approved in respect to style and intellectual content, is referred to you for judgment.

We have read this dissertation and recommend that it be approved.

$\begin{array}{r}\hline \text { Jeremy Pettit } \\ \hline \text { Milliam Kurtines } \\ \hline \text { Wendy K. Silverman, Major Professor Kenny }\end{array}$

Date of Defense: November 8, 2012

The dissertation of Maria Pienkowski is approved.

Dean Kenneth Furton

College of Arts and Sciences

Dean Lakshmi N. Reddi

University Graduate School

Florida International University, 2013 


\begin{abstract}
OF DISSERTATION
THE IMPACT OF MATERNAL ACCULTURATION, YOUTH AGE, SEX AND ANXIETY SENSITIVITY ON ANXIETY SYMPTOMS IN HISPANIC YOUTH

by
\end{abstract}

Maria Pienkowski

Florida International University

2013

Miami, FL

Professor Wendy K. Silverman, Major Professor

Despite progress that has been made in the areas of maternal acculturation and internalizing symptoms in Hispanic youth, much remains to be learned about the relation between maternal acculturation and youth anxiety. The inclusion of cognitive vulnerabilities such as anxiety sensitivity (AS) further adds to the understanding the development of anxiety in Hispanic youth. Examining the role that youth age and youth sex play in the relation between AS and youth anxiety symptoms also can further understanding of the development of youth anxiety. Thus, the specific aims of this dissertation were to examine whether: (1) a confirmatory factor analysis (CFA) would yield a two factor structure of the Stephenson Multigroup Acculturation Measure (SMAS; Stephenson, 2000); (2) maternal acculturation as measured by the SMAS is related to youth anxiety symptoms; (3) mother country of origin (i.e, Cuban or another Latin country) moderates the relation between youth AS and youth anxiety symptoms; (4) youth age moderates the relation between youth AS and youth anxiety 
symptoms; (5) youth sex moderates the relation between youth AS and youth anxiety symptoms.

In addition, research has shown Hispanic youth report more anxiety symptoms than non-Hispanic youth. The Revised Children's Manifest Anxiety Scale's (RCMAS; Reynolds \& Richmond, 1978) Lie Scale was included to examine whether it relates to Hispanic youths' reporting of anxiety symptoms in the current sample.

There were no significant differences in youth anxiety associated with the mother country of origin. Specifically, Cuban mothers and mothers from other Hispanic countries of origin did not significantly differ in their ratings of their child's anxiety symptoms. Mother country of origin did not moderate the relation between AS and youth anxiety symptoms. Also, no significant findings were found with respect to effects of age on the relation between anxiety sensitivity and anxiety. The study's main contributions and potential implications on theoretical, empirical, and clinical levels are further discussed. 


\section{TABLE OF CONTENTS}

CHAPTER

PAGE

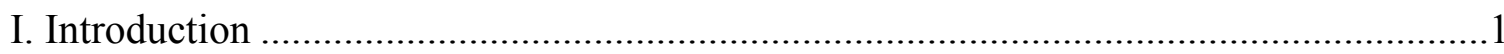

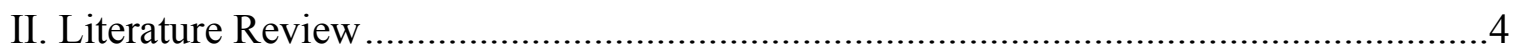

History of Acculturation as a Concept......................................... 5

Conceptualizing and Operationalizing Acculturation ..................................................

Limits of the Acculturation Categories Model ………..............................................

Acculturation Categories Model Hispanic Samples ...................................................10

The Influence of Hispanic Parent Acculturation........................................12

The Relation between Hispanic Youth Psychopathology and Acculturation as Indexed by Maternal Country of Origin ...............................................................13

Hispanics in Miami ........................................................ 15

Anxiety-Related Somatic Symptoms..........................................16

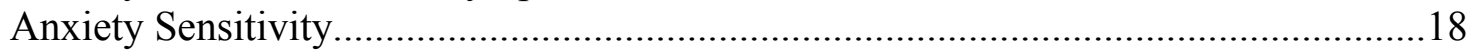

Anxiety Sensitivity in Anxiety-Disordered Youth............................19

Anxiety Sensitivity in Hispanic Youth ..................................................................20

The Relation Between Anxiety Sensitivity and Age in Hispanic Youth........................23

The Relation Between Anxiety Sensitivity and Sex in Hispanic Youth.........................28

Variability in Reporting of Anxiety Symptoms by Hispanic Youth...............................28

Purpose of the Present Dissertation Study ...............................................................29

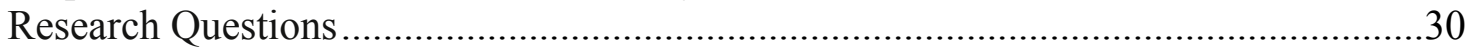

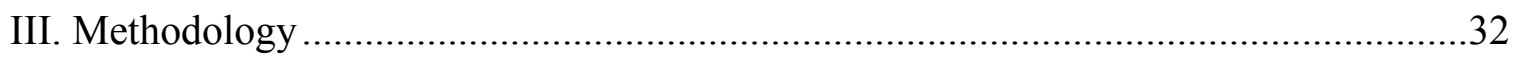

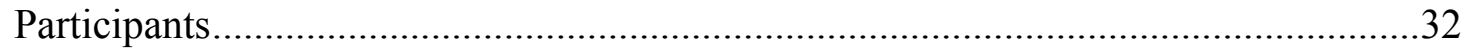

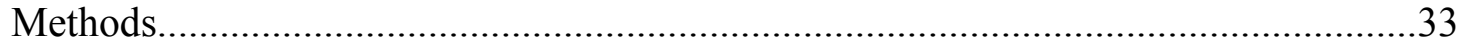

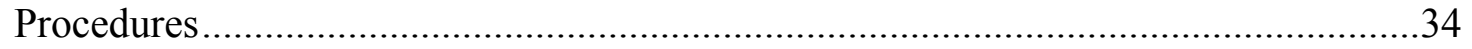

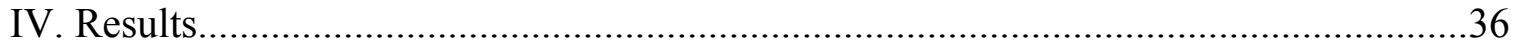

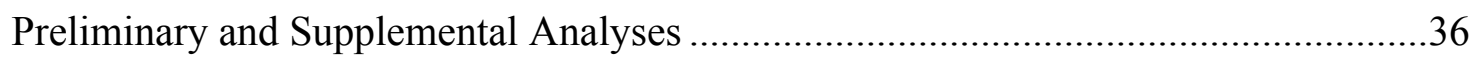

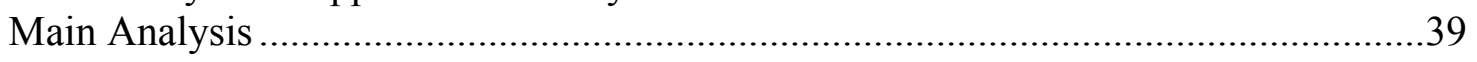

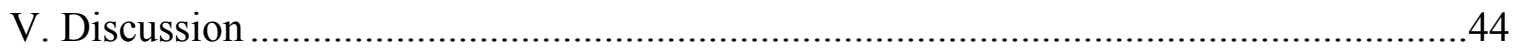

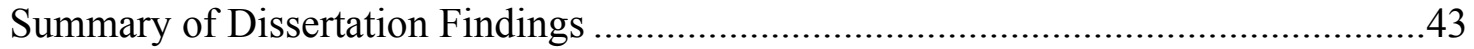

Contribution of the Present Study and Implications ....................................................4

Limitations and Future Research Directions.................................................................49

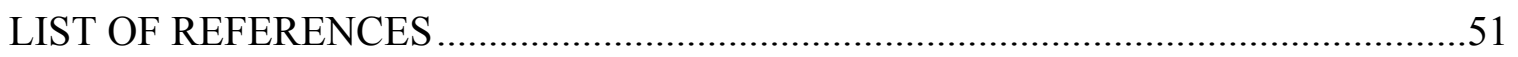

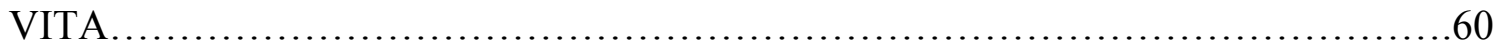




\section{Chapter I.}

\section{Introduction}

The dramatic increase in the Latino population across the United States is changing the face of the nation's youth. Currently, one in five public school children are Hispanic, compared to one in eight in the 1990s. By 2050, more Hispanic schoolage children than non-Hispanic white school-age children will reside in the United States (Fry \& Gonzales, 2008). Phobic and anxiety disorders are among the most common child and adolescent mental health problems with prevalence rates as high as $20.2 \%$ in community and $44.7 \%$ in clinic samples (Costello, Egger, Copeland, Erkanli, \& Angold, 2008). (From here on, children and adolescents are referred to as youth.)

Although no national data are available on the prevalence of anxiety disorders or anxiety-related problems among Hispanic youth, studies suggest that Hispanic youth experience significantly higher levels of anxiety than European Americans, but not African Americans (Glover, Pumariega, Holzer, Wise \& Rodriguez, 1999; Roberts, Roberts, Xing, 2006). Approximately 8\% of Mexican-American youth (age 11-17 years) experienced anxiety disorder in the past year (Roberts et al., 2006). As summarized by Gonzalez et al. (2009), Gonzalez et al. (2002), and UmañaTaylor, \& Alfaro (2009), most of the research on foreign-born and US-born Hispanic youth has examined how acculturation affects psychological well being. Acculturation is the process by which individuals are influenced by, and incorporate, norms, practices, behaviors, languages and customs of the host country (Warner, Valdez, Vega, de la Rosa, Turner, \& Canino, 2006). However, most of the work on acculturation, which has examined both parent and youth acculturation, focused on 
substance abuse and risky behaviors. There is scant research on how parental, specifically maternal acculturation is related to the psychological well being of Hispanic youth, especially in relation to anxiety symptoms. Much of the literature instead has focused on proxy measures of acculturation (e.g.,. mother language preference, parents' immigration status) rather than continuous measures that directly assessed acculturation.

Similarly, there is scant empirical information about other aspects of anxiety and its disorders in Hispanic youth, including anxiety sensitivity. Anxiety sensitivity (AS) is a cognitive vulnerability that refers to an individual's belief that his or her anxiety-related physical symptoms may have negative physical, social and psychological consequences (e.g., Reiss, 1991; Reiss, Peterson, Gursky, \& McNally, 1986). Anxiety sensitivity has been found to be elevated in samples of anxious youth relative to non-anxious samples, suggesting AS may play a role in the maintenance and development of anxiety disorders in youth (Weems, Hayward, Killen, \& Taylor, 2002). There are only two studies (Pina \& Silverman, 2004; Varela et al., 2007) that have addressed AS in Hispanic youth samples. The effects that youth age and youth sex have on Hispanic youth anxiety and AS also are not well represented in the current literature.

Lastly, studies conducted by Varela and colleagues ( Varela et al., 2004; Varela et al., 2008; Varela et al., 2007) show that Hispanics in the United States and Hispanics in their country of origin report more somatic symptoms and anxiety symptoms than their European American counterparts. Pina, Silverman, Saavedra, and Weems (2001) found Hispanic youth and their parents had significantly higher 
RCMAS-C Lie scale and RCMAS-P Lie scale scores, respectively, than European Americans. These results suggest that differences in the reporting of anxiety symptoms could be influenced by ethnicity (Varela et al., 2009).

In sum, there is need for research that addresses anxiety in Hispanic youth. The research needs to address the effects of maternal acculturation on the development of anxiety in Hispanic youth. It also needs to address the construct of AS and factors such as youth age and sex in Hispanic youth samples.

Providing empirical answers to the previously mentioned issues constituted the main objectives of this dissertation. Each of the objectives along with the relevant background information are elaborated upon in Chapter II of the dissertation. 


\section{Chapter II.}

\section{Literature Review}

The literature review begins first with a history of acculturation. Next, the conceptualization and operational definition of acculturation is elaborated upon within the context of Hispanic groups. Problems with the operational definition of acculturation also are addressed. The operational definition of acculturation is followed by a discussion of acculturation and its relation to anxiety in Hispanic groups. Emphasis is placed on the scarcity of empirical knowledge regarding the impact of maternal acculturation on youth anxiety and anxiety phenomenology including anxiety sensitivity(AS). AS as a cognitive vulnerability referring to an individual's belief that his or her anxiety related physical symptoms may have negative physical, social and psychological consequences, is also discussed (e.g., Reiss, 1991; Reiss, Peterson, Gursky, \& McNally, 1986).

The literature review next discusses the potential influence of youth ethnicity and AS. Emphasis is placed on the lack of empirical information about Hispanic youth and AS. The literature review next discusses AS and age. Inconsistencies found in the literature regarding the relation between AS and age also are explained. The explaination is followed by a discussion of sex and AS; the paucity of information in this area is addressed. Lastly, The Revised Children's Manifest Anxiety Scale's (RCMAS; Reynolds \& Richmond, 1978) Lie Scale was included to examine whether it relates to Hispanic youths' reporting of anxiety symptoms. The literature review concludes with a summary of the study's research questions and hypotheses. 


\section{History of Acculturation as a Concept}

The term "acculturation" has roots in archeology, where it appeared in the late 19th century writings of J. W. Powell (Rudmin, 2003). Upon examining Native American languages, Powell wrote: "The force of acculturation under the overwhelming presence of millions [of Europeans] has wrought great changes" (as cited by Rudmin, 2003, p. 11). In his writings, Powell was describing the changes in Native American languages that occurred as a result of the interaction between Native Americans and European Americans.

In 1936, Redfield, Linton, and Herskovits described acculturation as having two distinctive essential elements: continuous contact and subsequent changes. These two elements are central in defining acculturation and were supported by the Social

Science Research Convention (SSRC) in 1954. Therefore, acculturation is understood as a phenomenon resulting when two or more independent cultures continuously encounter each other. The continuous contact causes subsequent changes and adaptations for either or both cultures (Berry, 1997; Redfield, Linton, \& Herskovits, 1936).

According to Redfield et al. (1936) and SSRC (1954), there are three components essential to understanding the acculturation process. First, there are two or more cultures distinct in observable ways. For instance, there may be differences in language, customs, values, or ideals. Second, these cultures are in constant contact with each other. The interaction between the cultures is where the changes are able to take place. Finally, the constant contact between cultures fosters transformations and adaptations to each culture. 
Historically, the focus of acculturation theory and research has been on society as a whole (Sodowsky, Ming Lai, \& Plake, 1991). However, within the last few decades, prolific amounts of research have been conducted on the psychological effects of acculturation on the individual (Burnam, Hough, Karno, Escobar, \& Telles, 1987; Cabassa, 2003; Chun, Organista, \& Marín, 2003; Cuéllar, Harris, \& Jaso, 1980; Magaña, De la Roncha, Amsel, Fernandez, \& Rulnick, 1996; Sodowsky, Ming Lai, \& Plake, 1991). At the individual level, research focuses on the changes in the individual's modality of culture. Examinations are concerned with the individual's perceptions, ideologies, behaviors, language(s), values, and beliefs as that individual lives in a new environment.

As the acculturation literature has evolved, a few theories and models have developed to explain the acculturation progression. Two theoretical frameworks have dominated the literature of the acculturation process as it pertains to the individual: a unidimensional paradigm and a multidimensional paradigm (Cabassa, 2003; Berry, 1997; Sodowsky, Ming Lai, \& Plake, 1991). These competing theoretical frames have produced a substantial amount of research and debate (Cabassa, 2003).

The unidimensional paradigm maintains that acculturation flows along a single continuum of immersion (Cuéllar et al., 1980). At one end of the continuum is immersion into the culture of origin, while at the other end of the continuum is immersion into the dominant culture (Cuéllar et al., 1980). Interestingly the unidimensional progression only affects the acculturating group. There is an underlying assumption that the acculturating group does not influence the host culture. The unidimensional model also assumes that acculturation progresses in the direction 
of assimilation. Assimilation involves relinquishing customs, values, and beliefs of the culture of origin for those of the dominant culture. According to this model, there is not room for biculturalism or multiculturalism. As such, this model is simplistic and provides an incomplete conceptualization of this cultural phenomenon.

The bidimensional and multidimensional model conceptualize acculturation as entailing two distinct dimensions: (a) conformity to the dominant culture and (b) preservation of the culture of origin (Cabassa, 2003; Magaña, De la Roncha, Amsel, Fernandez, \& Rulnick, 1996), with the assumption that both are possible. The individual retains aspects of the culture of origin while also assimililating to the dominant culture.

Individuals are seen as having the capacity to value and maintain the culture of origin while simultaneously engaging in the host culture (Berry, 1997; Cabassa, 2003). The multidimensional construct is a cultural dialogue or bidirectional relationships, in which both the dominant or host culture and the culture of origin are simultaneously integrated to varying degrees. Berry's model of acculturation (1997) is further elaborated on in the following section.

\section{Conceptualizing and Operationalizing Acculturation}

In social science research, acculturation is conceptualized as the process by which individuals are influenced by, and incorporate norms, practices, behaviors, languages, and customs of the host country (Warner, Valdez, Vega, de la Rosa, Turner, \& Canino, 2006). Acculturation can potentially influence individuals' preferences and attitudes about what are deemed "normal" behaviors (Warner et al., 2006). Acculturation, as applied to individuals, refers to changes that occur when 
individuals come in to contact with culturally dissimilar people, groups, and social influences.

Acculturation research can be conceptualized from an individual or group perspective. Acculturation affects the psychology of the individual as well as changing cultural aspects of the group (Gibson, 2001). At the group level, the study of acculturation entails an understanding of the culture, institutions, and interactions among people. Within psychological research, more attention is placed on the acculturation experience of the individual, that is, on the individual's values, beliefs, and behaviors. Cabassa (2003) has noted that many measures of acculturation attempt to capture the individual's level or type of acculturation style. There is a degree of variability among acculturation experiences (Berry, 1997; Cabassa, 2003), which makes it a worthwhile endeavor to focus on the individual's acculturation style and the implications of such a style.

An influential approach to conceptualizing and operationalizing acculturation was proposed by Berry (1980). Berry's theory of cultural-acquisition proposed that when individuals move to a different country, they attain the beliefs, values, and practices of the receiving country (receiving culture acquisition). They also retain the beliefs, values, and practices of their mother country (heritage culture retention). Berry's cultural-acquisition theory casts receiving culture acquisition and heritage culture retention as two different dimensions. The intersection of these two dimensions creates four categories of acculturation: assimilation (adopts the receiving culture and discards the heritage culture), separation (rejects the receiving culture and retains the heritage culture), integration (adopts the receiving culture and attains the 
heritage culture), and marginalization (rejects both the receiving and heritage cultures) (Schwartz, Unger, Zamboanga, \& Szapocznik, 2010). Berry's integration category also has been referred to as biculturalism (Benet-Martinez \& Hariatos, 2005).

This multidimensional approach utilizes the constructs of assimilation and enculturation. Assimilation refers to Berry's (1980) category of adopting culturereceiving practices, values, and identifications, and ridding oneself of practices from the culture of origin. Enculturation encompasses the process of selectively obtaining or retaining elements of one's culture of origin while also acquiring elements of the receiving culture in which one lives (Weinreich, 2009). Several investigators have raised concerns with Berry's acculturation model, as summarized in the next section.

\section{Limits of the Acculturation Categories Model}

One major concern of Berry's (1980) acculturation model relates to the use of priori cut-off points and median split approaches. A priori cut-off points, such as the sample median (Giang \& Witting, 2006) or the midpoint on the range of possible scores as cut-off points (Coatsworth et al., 2005), have been the primary method of classifying individuals as either low or high in each category.

A second concern relates to the inclusion of a marginalization category in Berry's model (Rudmin, 2003, 2009). With regard to the use of a priori cut-off points and median splits, Berry's model creates a 2 x 2 matrix of acculturation categories that classifies individuals as either low or high on receiving-culture acquisition or heritageculture retention. The use of a priori cut-off points increases the chance that equal numbers of participants will be classified as low and high on each dimension. By using a priori cut-off points, all four of Berry's categories (high assimilation and 
enculturation; high assimilation and low enculturation; low assimilation and high enculturation; low assimilation and low enculturation) are represented in a given sample. The cut-off point between low and high on each category is arbitrary, however, and will differ across samples, which makes comparisons across studies challenging. Also, the use of a priori classification conventions assumes all four categories exist and are equally valid (Rumdin, 2003). Because different samples are likely to have different distributions and different cutoffs, participant characteristics in each of the four categories are sample specific.

The second concern regarding Berry's model of acculturation relates to the marginalization category of Berry's (1980) model. Individuals who fall into this category are low on both assimilation and enculturation. There is low likelihood however that individuals will develop a cultural sense of self without drawing on either the heritage or receiving cultural contexts (Berry, 2006). Given these concerns, researchers have taken steps to address them, as summarized next.

\section{Acculturation Categories Model in Hispanic Samples}

Schwartz and Zamboanga (2008) performed a latent class analysis to ascertain the extent to which acculturation orientation categories could be derived from continuous measures of heritage and receiving cultural practices. The sample was comprised of 436 Hispanic students enrolled at the University of Miami, FL. Schwartz and Zamboanga (2008) used the Stephenson Multigroup Acculturation Scale (SMAS; Stephenson, 2000) as the primary clustering variable. The SMAS assesses orientation towards heritage and American cultural practices in areas such as language, food, and entertainment. The Acculturation, Habits, and Interests Multicultural Scale for 
Adolescents (Unger et al., 2002) was used to validate the cluster solution. Specifically, participants were asked to identify whether their preference for performing the cultural practice indicated in each item most reflected the United States, their country of origin, both, or neither.

The results provided partial support for Berry's (1980) model in that a total of six, not four, classes emerged, suggesting Berry's categories may have multiple variants within a given sample. Also, one of the classes that emerged had a combination of the assimilation and integration categories. Of Berry's acculturation orientation categories, three emerged in the latent class analysis - separation, assimilation, and integration (biculturalism). However, the separation class also was characterized by some degree of biculturalism. Further, the previous marginalization class identified by Berry (1980) was not found (Schwartz \& Zamboanga, 2008).

The prominence of biculturalism identified by Schwartz and Zamboanga (2008) is consistent with other studies (e.g., Chia \& Costigan, 2006; Coatsworth et al., 2005; Pierce, Clark, \& Kaufman, 1978). Also, because Schwartz and Zamboanga's data were collected in Miami where biculturalism is strongly encouraged among youth (e.g., Schwartz, Pantin, et al., 2006; Stepick \& Stepick, 2002), biculturalism may have been even more prominent across Berry's acculturation orientation categories (i.e., separation, assimilation, integration) in this sample than others (e.g., Chia \& Costigan, 2006; Coatsworth et al., 2005; Pierce, Clark, \& Kaufman, 1978).

In sum, Schwartz and Zamboanga (2008) provide partial but not full support for Berry's model of acculturation (1980). Of Berry's four acculturation categories, three classes emerged from the latent class analysis -- separation, assimilation and 
integration (biculturalism). Unlike Berry's model (1980), no marginalization class

emerged and the separation class was characterized by some degree of biculturalism. Implications of these findings are discussed later in the dissertation.

There is a need to confirm Berry's model in the current dissertation sample using the SMAS (Stephenson, 2000) given that this is a widely used continuous measure of acculturation that has yet to be validated in a sample of Hispanic parents seeking services for their children.

The Influence of Hispanic Maternal Acculturation on Hispanic Youth

\section{Psychopathology}

Although research on immigrant families has shown parental acculturation is related to emotional well being of Hispanic youth, no study has examined whether a link exists between the mother's level of acculturation and youth anxiety. Studies have been conducted using proxy variables such as parent primary language choice (i.e., English, Spanish), parent ethnicity and parent nativity. However, there have not been any studies examining parent acculturation on youth anxiety using a continuous acculturation measure in Hispanic youth.

Two studies have produced evidence for a relation between mothers' levels of acculturation and youth depressive symptoms in community samples of MexicanAmerican mothers and their children (Dumka, Roosa, \& Jackson, 1997; Knight et al., 1994), but the two studies' findings were inconsistent. Knight et al. (1994) found high maternal acculturation levels predicted increased youth depressive symptoms; Dumka et al. (1997) found high maternal acculturation predicted decreased youth depressive symptoms. 
Gonzales, Knight, Morgan-Lopez, Saenz, and Sirolli (2002) suggested that these inconsistent findings could be associated with differences in mothers' acculturation levels, measured by the Acculturation Rating Scale for Mexican Americans (ARMSA; Cuellar, Harris, \& Jasso, 1980). Dumka et al.’s (1997) sample was predominately immigrant and unacculturated Mexican-American mothers; Knight et al.'s (1994) was limited predominately to highly acculturated mothers. Further, both studies examined youths' internalizing problems (i.e., depressive symptoms). The current dissertation study is the first to examine whether a relation exists between mother's level of acculturation and youth anxiety symptoms.

The Relation between Hispanic Youth Psychopathology and Acculturation as Indexed by Maternal Country of Origin. Alegria, Mulvaney-Day, Torres, Polo, Cao, and Canino (2007) used archival data from the National Latino and Asian American Study (NLAAS) to analyze prevalence rates of psychiatric disorders among Latino subgroups. A subsample of NLAAS consisted of 2554 English- and Spanish-speaking Latino adults from four major US Latino subgroups: Mexican ( $\mathrm{n}=868$ ); Puerto Rican $(\mathrm{n}=495)$; Cuban $(\mathrm{n}=577)$; and "other" $(\mathrm{n}=614)$. Results indicated that being born in the United States was associated with psychiatric diagnoses in the past year in each of the Latino subgroups. The results are consistent with the 'immigrant paradox,' or the concept that foreign nativity has a protective effect against psychiatric disorders. The above findings are relevant to the current study in that it draws attention to the increased prevalence rates of psychiatric disorders among Latino subgroups.

However, the 'immigrant paradox' has not been supported in other prevalence studies. For example, Alegria et al. (2008), using the same sample as Alegria et al. 
(2007), found the immigrant paradox to be true for Latinos when examined in aggregate. When Latinos were disaggregated by subgroups, the immigrant paradox finding was observed for Mexicans only. Mexican immigrants reported a significantly lower prevalence of major depressive episode, any depressive disorder, social phobia, any anxiety disorder, alcohol dependence and abuse, any substance disorder or any disorder than the other Latino subgroups (i.e., Puerto Rican, Cuban, Columbian). No protective effect was found for either the Cuban or other Latino subgroups except in the case of substance abuse disorders. Thus, it is unclear whether Cuban youth born in the US are at heightened risk for the development of psychopathology (Alergria, 2008).

In another study, effects of birthplace on internalizing symptoms were examined in a sample ( $\mathrm{N}=2,840)$ of Latino children (56.9 months to 79 months) from the Early Childhood Longitudinal Study-Kindergarten data set (Dawson \& Williams, 2008). Teachers rated significantly higher levels of internalizing symptoms items (i.e., anxiety, loneliness, low self-esteem, sadness) in children born in the US than in children not born in the US on the Social Rating Scale System (Gresham \& Elliot, 1990).

Dawson and Williams's (2008) findings are consistent with Alegria et al.'s (2007) in suggesting Hispanic youth born in the US may be at a greater risk for developing psychopathology than other minority groups. However, the study did not differentiate between Latino subgroups. Hence, it is unclear whether these findings hold only for Latinos when they are aggregated into one group. The current dissertation study therefore adds to the literature by differentiating between youth 
whose mothers are from Cuba from youth whose mothers are from another Latin country of origin (i.e. Puerto Rico, Mexico, Columbia, Nicaragua, Honudoras, Chile, Argentina, Spain, Brazil) in youth reported anxiety symptoms.

Hispanics in Miami While Hispanics are quickly becoming one of the largest minority groups in many cities in the US, Miami's Hispanic population is unique. Sixty-percent of the population of Miami and its surrounding area are Hispanic, with the largest subgroup of individuals being of Cuban-descent (Wang, Schwartz and Zamboanga, 2010).

Cuban-Americans are a unique subgroup of Hispanics in the United States in many ways. For example, they have the highest socioeconomic status of any Hispanic minority group. Cuban-Americans also have the largest retention of Spanish as their primary language (Portes \& Stepick, 1993). Also, the retention of strong family ties among Cuban Americans aid in the migration of new family members from Cuba to the US and the large Cuban community in Miami has made the transition for many Cubans less stressful than for some other Latino groups. United States government aid given to Cubans who resettle in the States has also been an important factor in their ease of adaptation (Grenier \& Stepick, 1992).

Studies of Cuban mental health report lower rates of distress and disorder as compared to other Hispanic subgroups. These lower rates of distress and disorder may be attributable to US government aid as well as the higher pre-migration social status of Cuban immigrants. While Cuban immigrants do experience emotional distress resulting from the separation of families between Cuba and the US as well as the great difficulties of returning to Cuba for important family transitions, epidemiological data 
do not provide evidence that such stressors lead to the development of mental health problems in the Miami Cuban population.

\section{Anxiety-Related Somatic Symptoms}

Somatic symptoms are key clinical features of anxiety disorders and play a significant role in anxious states (Beidel, Christ, \& Leung 1991; Last, 1991). High rates of somatic symptoms have been found in past studies of clinically anxious youth. For example, Last (1991) found 60\% of anxious youths reported "clinically significant" somatic symptoms.

Although youth with anxiety disorders and parents vary in their respective reports of youths' anxiety-related somatic symptoms, the symptoms most commonly reported by both sources are headaches, stomachaches, muscular tension and feeling jittery (Ginsburg, Riddle, \& Davies, 2006). Anxiety-related somatic symptoms can further lead to significant impairment in anxious youth, as well as being associated with excessive school absenteeism, frequent doctor's visits, and in extreme cases, visits to the emergency room. Research also indicates that anxiety-related somatic symptoms contribute unique variance to youth functioning such as academic performance (Hughs et al., 2007). Additionally, research indicates that youth with anxiety disorders exhibit more frequent and a greater number of anxiety-related somatic symptoms than youth from community samples (Dorn et al., 2003).

The notion that individuals who suffer from emotional distress including anxiety may express their distress somatically has been researched extensively in the clinical literature for well over two decades (Biedel et al., 1991, Last, 1991). The literature is relatively scant, however, when it comes to developing and testing 
theoretical perspectives aimed at advancing understanding regarding how anxietyrelated somatic symptoms come about in anxious individuals.

Anxiety-Related Somatic Symptoms in Hispanic Youth. In community samples, Hispanic youth have been found to report significantly more anxiety-related somatic symptoms than their European-American counterparts. Varela, Vernberg, SanchezSosa Riveros, Mitchell, and Mashunkashey (2004) found in a community sample of Hispanic youth (ages 10-14 years) that after controlling for socioeconomic status (SES) and scores on the RCMAS (Reynolds \& Richmond, 1978) Lie scale, Mexican $(\mathrm{n}=53)$ and Mexican American $(\mathrm{n}=50)$ youth reported more anxiety-related somatic symptoms on the RCMAS Physiological subscale and more symptoms on the Children's Anxiety Sensitivity Index (CASI; Silverman, Fleisig, Rabian, \& Peterson, 1991) than European American youth ( $\mathrm{n}=51)$. In addition, the Mexican American youths reported more anxiety than the Mexican youths only when it came to ratings of anxiety-related somatic symptoms on the RCMAS Physiological subscale or symptoms on the CASI (Varela \& Henlsey-Maloney, 2009). Mexican youth reported more anxiety-related somatic symptoms than European American youth.

In another study using a sample of Columbian youth residing in Columbia $(\mathrm{n}=163)$ and the United States ( $\mathrm{n}=116)$, as well as white non-Latino youth $(\mathrm{n}=72)$, Varela, Weems, Berman, Hensley, and Rodriguez de Bernal (2007) found Colombian and Latin American youth reported more anxiety-related somatic symptoms on the Brief Symptom Inventory (BSI, Derogatis, 2000) than white non-Latino youth. Also, Latino and Colombian females reported more anxiety symptoms than white nonLatino males on the BSI. 
These two studies by Varela et al. $(2004,2007)$ are consistent in showing that Hispanic youth report more anxiety-related somatic symptoms than white nonHispanic youth. Despite the importance of the Varela et al. findings, their generalizability to other groups of Hispanic youth in the United States is unclear. As discussed in the following section, there is little evidence examining how anxietyrelated somatic symptoms are related to anxiety in Hispanic youth. Examining the contribution of cognitive vulnerabilities like AS to the maintenance and development of anxiety symptoms in Hispanic youth could further clarify anxiety phenomenology in Hispanic youth. Anxiety Sensitivity

Anxiety sensitivity (AS) is a cognitive vulnerability that refers to an individual's belief that his or her anxiety-related somatic symptoms may have negative psychological, physical and social consequences (e.g., Reiss, 1991; Reiss, Peterson, Gursky, \& McNally, 1986). Psychologically, the physical sensations give the individual the sense that they are going crazy or losing control. Physically, those with high AS believe that the physical sensations they experience are harmful and will inevitably lead to catastrophic outcomes. Socially, those with high AS worry that others will noticed the increased anxious arousal. In turn, they will be criticized, ridiculed, rejected or socially isolated (Walsh, Stewart, McLaughlin, \& Comeau, 2004).

Originally, the concept of anxiety sensitivity was derived from Reiss' expectancy theory (1991). Expectancy theory perports that a set of fundamental fears, including AS, amplify fear, anxiety and panic. These fundamental fears qualitatively 
differ from more common fears, like animal fears or situational fears. The two major differences are: (1) fundamental fears are logical reductions of common fears, and (2) fundamental fears are commonly aversive to people in general. AS was considered an "amplification factor." That is, differences in levels of AS could explain differences among individuals in the conditioning of fear and consequences to fear (Taylor and Federoff, 1999). These individual differences are thought to result from variations in genes that make one more prone to anxiety. Similarly, cognitive factors can also lead to the formation of beliefs that anxiety symptoms will lead to negative consequences (Reiss, Silverman, \& Weems, 2001).

Anxiety sensitivity has been found to be elevated in samples of anxious youth relative to non-anxious samples, suggesting AS may play a role in the maintenance and development of anxiety disorders in youth (Weems, Hawyard, Killen, \& Taylor, 2002). Further research into anxiety sensitivity could elucidate other mechanisms of anxiety sensitivity in youth.

Anxiety Sensitivity in Anxiety-Disordered Youth

Noel and Francis (2011) conducted a meta-analysis of the role of child AS in child anxiety. The inclusion criteria for the studies were (a) published in a peer reviewed, English language journal, (b) the age of participants was between 6 and 18 years, (c) the AS outcome measures were one of the following, the CASI ( Silverman et al.1991), Anxiety Sensitivity Index (ASI; Reiss et al. 1996), Anxiety Sensitivity Index for Children (ASIC; Laurent \& Stark, 1993), or Childhood Anxiety Sensitivity IndexRevised (CASI-R; Muris 2002), and (d) the informants were the child participants themselves. Exclusionary criteria were (a) provided insufficient data for the 
calculation of effect size (and this information could not be obtained from the author) and (b) used the same participant pool as a previously published study and conducted identical analyses.

One of the key questions the study addressed was whether AS could distinguish clinic referred youth with anxiety disorders from non-clinic referred youth without anxiety disorders. Five studies (Anderson \& Hope, 2009; Hayward et al., 2003; Laurent \& Stark, 1993; Silverman et al., 2003; Vasey et al., 1995) were included in the calculation of the weighted mean effect size. In each of these five studies, the comparison groups were comprised of anxiety-disordered youth and nonclinical youth. The analysis revealed that youth diagnosed with an anxiety disorder demonstrated higher AS than non-clinical youth ( $\mathrm{d}=0.64)$, with non-significant variability in the findings across the five studies (Noel \& Francis, 2011). These results indicate that in a sample of anxiety-disordered youth, AS and anxiety disorders are related. Anxiety Sensitivity in Hispanic Youth.

Varela et al. (2007) examined the role youth sex, AS, and ethnic minority status plays in the expression of internalizing symptoms in a community sample of Latin American adolescents ( $\mathrm{n}=116)$, European American adolescents $(\mathrm{n}=72)$, and Columbian adolescents in Columbia ( $\mathrm{n}=163)$. Given culture defined gender role expectations, the authors hypothesized that gender would amplify the relation between AS and internalizing symptoms.

The results revealed that AS and being female predicted anxiety and depression symptoms independent of cultural background. Youth sex (i.e., being female) uniquely predicted anxiety and depression symptoms independent of ethnicity. 
To date, there have not been any studies examining the role that youth sex and ethnicity have on the relation between AS and anxiety in clinic-referred Hispanic youth.

Pina and Silverman (2004) is the only study to examine youths' somatic symptoms and AS in a sample drawn from the same population as the proposed study (i.e., South Florida, which contains a large proportion of Cuban-Americans). In a sample of 152 Hispanic youth who presented to an anxiety disorders specialty clinic, Pina and Silverman (2004) assessed youths' anxiety-related somatic symptoms and youths' anxiety associated with these somatic symptoms (i.e., AS; Reiss, 1991). All youth met DSM-IV criteria for a primary anxiety disorder using the Anxiety Disorders Interview Schedule - Child and Parent Versions (Silverman \& Albano, 1996).

Results indicated that youths' levels of distress associated with their somatic symptoms varied by the youths' ethnocultural group (i.e., Cuban-American, nonCuban-American/Hispanic-Latino) and language of choice (English, Spanish). Specifically, parents of youth in the Cuban-American (CA) and European American ethnocultural groups reported significantly less somatic symptoms (as reported on the Child Behavior Checklist somatic subscale) than parents of youth in the non CubanAmerican/Hispanic-Latino groups (non-CA/HL). Within the group of youth whose parent's language of preference was English, CA youth reported somatic symptoms significantly less distressing (using the CASI total scale and CASI Disease Concern subscale) than non-CA/HL youths. However, within the Spanish language preference group, CA youth reported somatic symptoms significantly more distressing than non- 
$\mathrm{CA} / \mathrm{HL}$ using these same scales. The results also highlight the importance of the relationship of somatic symptoms to AS in Hispanic youth.

These results suggest that Hispanic youths' distress about somatic symptoms may be an important aspect of the clinical phenomenology of Hispanic/Latino youths with anxiety disorders. These results are theoretically significant as they indicate that Hispanic youth from different countries of origin (i.e., Mexico, Columbia, Cuba) report more somatic symptoms than their European American counterparts.

There is scant research on AS in Hispanic youth. Only two studies, Pina and Silverman (2004) and Varela et al. (2007) use Hispanic samples to examine AS in Hispanic youth. Silverman et al. (2003) used the results of past factor analytic studies of the Childhood Anxiety Sensitivity Index (CASI, Silverman, Fleisig, Rabian, \& Peterson, 1991) and the Anxiety Sensitivity Index (Reiss, 1983) to formulate hypotheses about factor models of AS. One of the samples used was a clinic sample of 258 children from the Child Anxiety and Phobia Program at FIU, which is the same program from which the current study drew its sample. The results provided strong support for a hierarchical model for AS as represented by the CASI with four facets: Disease Concerns, Unsteady Concerns, Mental Incapacitation Concerns, and Social Concerns. The Disease Concerns subscale contains items such as "it scares me when I feel shaky," "it scares me when my heart beats fast," when my heart beats fast I worry something is wrong," and "when my stomach hurts I worry that I'm really sick. Clinic referred youth with anxiety disorders had significantly higher scores than the following groups: youth's pooled non-clinic referred samples, clinic-referred youth with specific phobias; and the remaining clinic-referred youth. 
The Relation Between Anxiety Sensitivity and Age in Hispanic Youth AS in youth has been demonstrated to be uniquely predictive of youths' anxiety symptoms, thereby suggesting AS is an important risk factor in the development of anxiety and its disorders (Joiner et al., 2002; Silverman et al., 1991). However, a salient theme in the early research on youth AS was whether children and adolescents can both experience AS or whether AS requires a level of cognitive development for it to be exhibited uniquely from anxiety.

Chorpita, Albano, and Barlow (1996) suggested that children between the ages of 6 and 11 years old, who are thought to be in the Piagetian concrete-operational period of cognitive development, may lack the cognitive capacity to associate present internal sensations with future oriented consequences, especially if the consequences are abstract in nature (Noel \& Francis, 2011). Even while the question of whether AS was distinct from trait anxiety was being evaluated in the adult literature (McNally, 1989; Marian \& McNally, 1996); there were still questions as to whether AS (as measured by the CASI) and trait anxiety were distinct constructs for children and adolescents, particularly for younger children (Chorpita, Albano, \& Barlow, 1996). Chorpita et al. (1996) examined the incremental validity of the CASI with a clinic sample of youth diagnosed with an anxiety disorder ( $\mathrm{N}=112$, ages 7 to 17$)$. The authors used multiple regressions to examine the ability of the CASI to predict fear scores as measured by the FSSC-R and trait anxiety scores, as measured by the Trait subscale of the State- Trait Anxiety Inventory for Children (STAIC-T; Spielberger, 1973). Using two separate regression models, FSSC-R and STAIC-T scores were regressed onto age, CASI scores, the product term of CASI and age squared, and the 
Physiological Symptoms subscale (RCMAS-P; Reynolds \& Richmond, 1978) of the Revised Children's Manifest Anxiety Scale (RCMAS). In both models, the authors found the interaction term to be significant indicating that the incremental validity of the CASI increased with age in a curvilinear fashion. That is, the youth in the sample increased in age, the CASI became more valid.

Next, the authors split the sample into two groups, younger ( $n=43$, ages 7 to 11) and older ( $\mathrm{n}=69$, ages 12 to 17). Hierarchical multiple regression analyses were used to predict STAIC-T scores from CASI scores and either FSSC-R scores or RCMAS scores. Results indicated that the CASI only predicted additional variance for the older sample. Though the results support the idea that AS predicts trait anxiety in older youth, the authors interpret the results as the indicating that the CASI may not provide and accurate measure of AS when used with younger children.

However, Weems, Hammond-Laurence, Silverman, and Ginsburg (1998) showed, using the same measures as Chorpita et al. (1996) but using a more appropriate analytic strategy, that AS did contribute uniquely to predicting trait anxiety in children aged 6-11 years. The CASI scores in children predicted trait anxiety, suggesting a developmental trajectory of AS (Noel \& Francis, 2011). Weems et al. (1998) addressed several difficulties evident in the conclusions presented by Chorpita et al. (1996). Weems et al. noted that the issue raised by Lilienfeld (1989) in the adult literature was not whether AS predicted trait anxiety, but whether AS predicted additional variance beyond trait anxiety. The methodology employed by Chorpita et al. did not address this issue. Also, the small sample sizes used by Chorpita et al. may not have had enough predictive power to detect significant effects. 
Weems et al. sought to elucidate the issue of small sample size in Chorpita et al. (1998) by conducting various multiple regression analyses on a clinic sample of 280 youth (ages 6 to $17, M=10$ years) diagnosed with a primary anxiety disorder. As in Chorpita et al. (1998), the sample was split into two age groups, younger (ages 6 to 11) and older (ages 12 to 17). Scores on the STAIC-T were regressed hierarchically onto either FSSC-R or RCMAS-P scores in step 1, then, CASI scores in step 2. Scores on the CASI predicted additional variance in trait anxiety for younger, $\Delta \mathrm{R} 2=.16$ and .12 , and older children, $\Delta \mathrm{R} 2=.09$ and .16 , for the models using the FSSCR and RCMAS-P. To test the hypothesis that CASI would predict additional variance in fear scores beyond trait anxiety in both all youth, FSSC-R scores were regressed hierarchically onto STAIC-T scores, CASI scores and frequency of anxiety symptoms. The CASI was found to predict additional variance in fear beyond trait anxiety and anxiety, $\Delta R 2=.10$ and .12 , for both samples.

To test whether age moderated the prediction of trait anxiety from CASI scores, Weems et al. (1998) also conducted separate multiple regression analyses predicting STAIC-T scores, from CASI scores, FSSC-R scores, age and either the product term of age and CASI (to test for linear moderation) or age-squared and CASI (to test for curvilinear moderation). Neither interaction term was found to be significant. Finally, Weems et al. (1998) listed the partial correlations between FSSC$\mathrm{R}$ and CASI scores, as well as the predicted covariate-adjusted slope of the CASI on FSSC-R scores by age, controlling for STAIC-T scores in both analyses. Again, results did not indicate any significant results with respect to age. Taken together, 
these results confirm that the CASI exhibits incremental validity over trait anxiety in youth.

Noel and Francis (2011) used weighted mean effect sizes to address the both the unique correlational relationship between AS and anxious symptoms in children and the unique correlational relationship and AS in adolescents. With respect to the relation between AS and anxiety symptoms in children, Noel and Francis (2011) used five studies in the analysis (see meta-analysis, Noel \& Francis, 2011 for table). The results revealed that high levels of anxious symptoms were associated with high levels of AS after controlling for depression, child sex and physiological arousal. A small significant effect size was observed for this relation $(r=0.26, p<.001)$.

Noel and Francis (2011) also meta-analyzed eight studies to examine the relation between AS and anxiety in adolescents (see Noel \& Francis 2011 for studies cited). In these studies, a robust significant medium effect size was found. The medium effect size result indicates that the presence of more anxious symptoms is associated with higher levels of AS in adolescents after also controlling for depression, sex and physiological arousal. Since significant variability was observed between the findings of the eight studies, moderator analyses were performed to investigate whether a moderating variable could explain the variability across the studies included. Measures of AS (ASI, CASI, CASI-R, ASIC) and sample type (clinical, non-clinical) were tested as potential moderators. Moderation analysis indicated studies using CASI-R had a larger effect size compared to the studies using the other measures (ASI, CASI, ASIC). 
Lastly, a $z$-test was done to test if the overall correlation between AS and anxiety in children differed significantly from the overall correlation between AS and anxiety in adolescents. The correlation between AS and anxiety was significantly higher in adolescents than children. The results suggest that anxiety increases with age (Noel \& Francis, 2011). In comparing the FSN scores in the child analysis (FSN=31) to the adolescent analysis ( $\mathrm{FSN}=613$ ), there is a noticeable trend among four of the five studies reviewed above indicating that AS is a construct separate from anxiety. However, the lower FSN score in the child studies indicates a need for additional research on the relation between AS and anxiety in children under 12 years old (Noel \& Francis, 2011).

With regard to the role ethnicity plays in the relation between anxiety and AS, age seems to have an effect among reported anxiety symptoms in Hispanic youth. Silverman \& Ginsburg (1996) found that younger children report more fears on the FSSC-R than older children. However, there are no studies that have examined the interaction of age and ethnicity in Hispanic youth and how this interaction affects the relation between anxiety and AS.

Anxiety sensitivity and whether younger youth experience AS is a salient issue in terms of AS as a developmental construct. While the current literature (i.e. Weems et. al, 1998; Noel \& Francis, 2011) indicates that it is possible for younger youth to experiences AS, further validation of the relationship is needed, especially in Hispanic youth samples. Similar to the Weems et al. (1998), the present dissertation study used a Hispanic sample to examine how age affects the relation between anxiety and AS. 
The Relation Between Anxiety Sensitivity and Sex in Hispanic Youth There are several studies in the literature regarding AS and youth sex, which have yielded results suggesting that girls and boys may experience AS differently (e.g., Muris, Schmidt, Merckelbach, \& Schouten, 2001; Silverman, Ginsburg, \& Goedhart, 1999; van Windenfelt, Siebelink, Goedhardt,. \& Treffers, 2002). However, little work has been conducted on whether there are AS sex differences in Hispanic youth. In the only study to date, Varela et al. (2007) (summarized above) found high AS and being female predicted anxiety and depression symptoms independent of cultural background.

Variability in Reporting of Anxiety Symptoms by Hispanic Youth

Studies (Varela et al., 2004; Varela et al., 2008; Varela et al., 2007) show a pattern of results indicating that Hispanics in the United States and Hispanics in their country of origin report more somatic symptoms than their European American counterparts in the United States. However, the finding by Pina and Silverman (2004) showing that Hispanics of Cuban descent differed from Latinos of non-Cuban descent in their reporting of anxiety-related somatic symptoms draws attention to the importance of discerning differences between Hispanic groups in the United States. Varela et al. (2008) also found that Hispanics from Central America and Hispanics from other countries descent did not differ in physiological or anxiety scores. In sum, these results suggest that differences in the reporting of anxiety symptoms could be culturally influenced (Varela et al., 2009).

Given that Hispanic youth report more anxiety symptoms than non-Hispanic youth, further insight into differences in reporting of anxiety symptoms by Hispanic 
youth is warranted. Pina, Silverman, Saavedra, and Weems (2001) examined Lie Scale from the RCMAS and RCMAS-P scores in relation to youth age, ethnicity and Total Anxiety scores in a clinic-referred sample of 284 youth and their parents. In terms of between group differences in ethnicity, the results indicated that Hispanic youth and their parents had significantly higher RCMAS and RCMAS-P Lie scale scores than European Americans. The difference in the Lie Scale scores could suggest a difference in cultural norms between Hispanic and European American families. For example, the child's endorsement of items such as "I like everyone I know" or "My child likes everyone he/she knows" could reflect collectivistic qualities of Hispanic culture as opposed to being an indicator of defensiveness on the part of the participants.

\section{Purpose of the Present Dissertation Study}

The present dissertation study aimed to address three salient issues relating to Hispanic youth with anxiety disorders. First, the study addressed how maternal acculturation as measured with the SMAS (Stephenson, 2000) influences youth anxiety

and AS. Also, by using mother country of origin as a way to differentiate among the Hispanic subgroups, the present study addressed the lack of empirical knowledge regarding differences youth anxiety symptoms and AS in Hispanic subgroups.

Second, the dissertation study addressed how youth sex and youth age influenced the relation between AS and anxiety symptoms. The use of a clinicreferred anxious sample of Hispanic adds to current empirical knowledge in that only one study (Pina \& Silverman, 2004) evaluated AS in a similar sample. Further empirical evidence is necessary to elucidate how sex and age are related to AS. 
The present study did not directly identify possible culture mechanisms for Hispanic youths' over reporting of anxiety symptoms. However, it attempted to further the evidence towards discerning differences in the reporting of anxiety symptoms in Hispanic youth. Similar to Pina et al. (2001), the RCMAS Lie Scale was used in a sample clinic-referred, anxious sample of Hispanic youth.

\section{Research Questions}

The specific aims of this dissertation were to evaluate the following research questions and related hypotheses:

1. Will a confirmatory factor analysis (CFA) of the SMAS (Stephenson, 2000) in the current sample yield a two-factor solution?

Hypothesis 1: A CFA of the SMAS in the current sample will yield a two factor solution.

2. Is parent acculturation as measured by the SMAS (Stephenson, 2000) associated with youth anxiety?

Hypothesis 2: Parent acculturation is associated with youth anxiety. That is, those youth whose parents' are less acculturated will report less anxiety symptoms.

3. Does mother country of origin (i.e., Cuban or another Latin country) moderate the relation between youth anxiety sensitivity and youth anxiety?

Hypothesis 3: Youth whose mothers are from other Hispanic countries moderate the relation between youth AS and youth anxiety more than those whose mothers are from Cuba.

4. Does youth age moderate the relation between youth AS and youth anxiety in a Hispanic sample? 
Hypothesis 4: Youth age will moderate the relation between youth anxiety sensitivity and youth anxiety in a Hispanic sample.

5. Does youth sex moderate the relationship between youth AS and youth anxiety in a Hispanic sample?

Hypothesis 5: Youth sex will moderate the relationship between youth AS and youth anxiety in a Hispanic sample.

6. Will the RCMAS Lie Scale scores be predictive of youth anxiety symptoms?

Hypothesis 6: The RCMAS Lie Scale will be predictive of youth anxiety symptoms. 


\section{Chapter III}

\section{Methodology}

\section{Participants}

The present study consisted of an archival data set obtained from 604 youths (ages 6 to $16, \mathrm{M}=10.23, \mathrm{SD}=2.21$ ) and their mothers who presented to the Child Anxiety and Phobia Program (CAPP) at Florida International University (FIU). CAPP is a research clinic specializing in the treatment of youth anxiety disorders. All participants were referred to CAPP by school counselors, pediatricians, and other mental health professionals or by self-referral for difficulties due to excessive anxiety. Before coming to the clinic for evaluation, the parent completes a brief evaluation over the phone in order to ascertain if anxiety present. The participants' age range in the current study was similar to those of past clinical trials (Barrett et al., 1998; Kendall, 1994). The data analyzed in this study included all those who were evaluated at CAPP.

Sociodemographic information of the study's participants is provided in Table 1. As shown, $75 \%$ of the participants were Hispanic/Latino Americans, and 54\% were boys. The youths' age range of 6 to 16 years reflected the modal age range of the age of onset of separation anxiety disorder (SAD), social phobia (SOP), specific phobia, (SP), and generalized anxiety disorders (GAD) in the population and is reflective of the CAPP's referral patterns. For the purposes of this study, the Hispanic subsample of the larger sample was used. 


\section{Measures}

Distress or Aversiveness of Somatic/Physiological Symptoms

The main outcome measure that was used in the present study was the Childhood Anxiety Sensitivity Inventory (CASI; Silverman, 1991). The CASI consists of 18 items that assess the extent to which children and adolescents view the experience of anxiety-related somatic/physiological symptoms as distressing or aversive. Each item is rated using a 3-point scale as 1 (none), 2 (some), or 3 (a lot). Silverman et al. (1991) reported an alpha coefficient of 0.87 and test-retest re- liability estimates of 0.76 with a similar clinical sample (Pina \& Silverman, 2004). The CASI Total score will be used in this study. Examples of items from the CASI include "It scares me when I feel like I am going to faint" and "It scares me when I have trouble getting my breath."

In addition to using the CASI Total score, recent factor analytic studies show four facets of anxiety sensitivity contained on the scale: Disease Concerns, Unsteady Concerns, Mental Illness Concerns, and Social Concerns (Silverman et al., 2003). Anxiety Measures

The Children's Manifest Anxiety Scale - Revised (RCMAS; Reynolds \& Richmond, 1978) Total Score was used to measure youths' anxiety symptoms. Factor analytic studies have been generally consistent in reporting a three-factor structure (Worry/Oversensitivity, Social Concerns/Concentration, and Physiological) for the RCMAS Total Anxiety scale plus a lie scale (Reynolds \& Paget, 1981; Reynolds \& Richmond, 1979; Scholwinski \& Reynolds, 1985). The Lie Scale of the RCMAS is often used as an indicator of social desirability (Dadds, Perrin, \& Yule, 1998; 
Reynolds \& Richmond, 1985) and/or defensiveness (Joiner, 1996; Joiner, Schmidt, \& Barnett, 1996a; Joiner, Schmidt, \& Schmidt, 1996b). The Lie scale was used to ascertain possible reporting differences in youth AS and youth anxiety in the current sample.

Parent Acculturation

Stephenson Multigroup Acculturation Scale (SMAS; Stephenson, 2000). The SMAS consists of 32 items that assess behavioral and attitudinal aspects of acculturation that can be applied across ethnic groups and were administered to the mothers in the current study. The SMAS is scored according to two subscales: Ethnic Society Immersion (ESI) and Dominant Society Immersion (DSI). The ESI scale score assesses the level to which an individual maintains the values and practices of an ethnic group other than the dominant society and includes items such as, "I speak my native language at home.” The DSI scale score assesses the level to which an individual adopts the values and practices of the dominant society and includes items such as, "I think in English." Responses to each item are based on a 4-point Likert response format including: $1=$ True, $2=$ Partly true, $3=$ Partly false, and $4=$ False . Scores are determined by calculating mean item responses and range from 1 to 4 . Higher scores on ESI reflect lower acculturation while higher scores on DSI reflect higher acculturation. The alpha coefficients for the ESI and DSI subscales in this sample were .83 and .87 , respectively.

\section{Procedures}

Assessment interviews and questionnaires were administered after parental informed consent/child assent was obtained. All assessment interviews and 
questionnaires were generally completed in one session and conducted by doctoral level psychology graduate students. 


\section{Chapter IV}

\section{Results}

\section{Preliminary and Supplemental Analyses}

Outlier. Analyses were pursued using both non-model based and model-based methods. With respect to the former, multivariate outliers were identified by calculating leverage indices for each respondent. A leverage score four times greater than the mean leverage was defined as an outlier. There were no outliers found in the data using this approach. A model-based outlier analysis was also conducted. The model-based method involved regressing a randomly selected indicator for each endogenous variable onto an indicator for variables of which the endogenous variable was assumed to be a linear function. The regression analysis uses ordinary least squares (OLS) regression in a limited information estimation framework. Standardized df beta estimates were then examined for the predictors and intercepts for each case. Outliers were defined as the absolute value of standardized df beta scores in the excess of 1.0. No outliers were evident in the data using this approach.

Non-Normality. Univariate indices of skewness and kurtosis were examined to determine whether the absolute value of any of these indices is greater than 2.0. Nonnormality was evident in several variables in this study. The decision was thus to pursue structural equation modeling (SEM) analyses in Mplus 6 (Muthén \& Muthén, 2007) by using an estimator (MLR) robust to violations of normality based on the Huber-White algorithm (Huber, 1967; White, 1980).

Missing data. In the analysis of missing data, it was first determined whether there was a systematic bias in the patterning of missing data. The analysis involved 
computing a dummy variable reflecting the presence or absence of missing data for a given measure in the data. This dummy variable was then correlated with all other variables in the model as well as an array of demographic variables. No significant correlations were observed, indicating that there was no evidence of bias resulting from missing data.

Statistical power and sample size considerations. SEM requires taking into account statistical power, issues of the stability of the covariance matrix, and the use of asymptotic theory. With regard to statistical power, however, it is difficult to evaluate the power associated with specific path coefficients in complex SEM models because of the large number of assumptions about population parameters that must be made. With regard to the asymptotic theory and covariance stability, simulation studies suggest that sample sizes of 100 to 125 or larger often yield adequate results given that reasonably reliable measures are used (reliabilities greater than 0.65 ) and with a reasonable number of indicators per latent variable (Jackson, 2003; Jaccard \& Wan, 1996). The sample size of 604 participants along with psychometrically sound measures used in the present study exceeds this standard.

Structural equation modeling SEM. To explore the proposed models in this study, the data were analyzed using SEM in Mplus 6. Structural equation modeling is a powerful and flexible tool that, besides assessing the directional and non-directional relationships among observed and unobserved variables, recognizes the imperfect nature of the measurement and thus explicitly specifies error terms for all the measurements in a model (Byrne, 2001). Such specifications are important because traditional multivariate methods like regression assume variables in the analysis are 
perfectly reliable or free of measurement error. Additionally, SEM provides flexibility, more so than alternative approaches (e.g., regression analysis), because it does not require that a variable be either a cause or an effect of another variable. Finally, SEM affords formal significance testing of competing conceptual models to identify those models that are the most appropriate given the data (Byrne, 2001). The data were first analyzed using a limited information framework where the relationship between each of the possible moderators and youth anxiety was tested (youth sex, youth age, country of origin, ESI, DSI, AS, and RCMAS Lie scale).

Fit indices. Model fit was evaluated with the comparative fit index (CFI) of values $\geq 0.95$, the root-mean-square error of approximation (RMSEA) of values $\leq$ 0.08 , and the $p$ value for close fit test of values $\geq 0.05$ representing acceptable fit (Bentler, 1990; Brown \& Cudeck, 1993). More focused fit indices were also examined with modification indices of values $\leq 3.83$ and standardized residual values of $\leq 1.96$ representing acceptable fit.

\section{Confirmatory Factor Analysis of the SMAS}

A confirmatory factor analysis (CFA) was performed to assess the factor structure of the SMAS (Stephenson, 2000). The items in the SMAS are considered categorical variables. On the two factors, two items $(12,25)$ double loaded on the ESI and DSI. The two items that did not load were left in the model as theoretically they could load onto either ESI or DSI subscales. One item (4) did not load on either factor (see Table 1) because it was not statistically significant ( $>>05)$. Therefore was trimmed from the model. 
Even though the fit standards for the $\chi^{2} / \mathrm{df}$ ratio (i.e., under 3.00 ) were not obtained, the fit of the 2 factor model was satisfactory by the other criterion: $\chi^{2}$ (403) $=1974.579, \mathrm{CFI}=0.996$ (i.e., greater than .95), TLI $=0.995$ (i.e., greater than .95), $\mathrm{SRMR}=0.018$ (i.e., less than .08), and RMSEA=.070 (i.e., less than .08).

\section{Main Analysis}

Model Tests

Structural equation modeling analysis was performed to test research questions. Two models are defined (Table 4). Model 2 is related to Model 1 as follows: In Model 2, the path between AS and depression was fixed to zero, because it was nonsignificant in the full model.

Fit indices for each of the two models appear in Table 5. To begin the nested models analysis, the full model (Model 1) was tested for model fit. Even though the

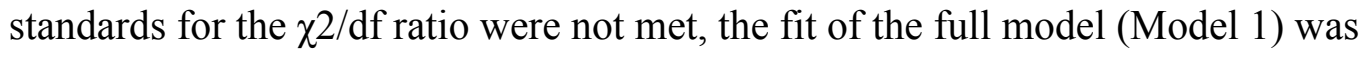
satisfactory: $\chi 2(11=67.869), \mathrm{CFI}=0.975$ (i.e., greater than .95$), \mathrm{TLI}=0.944$ (i.e., greater than .90), RMSEA $=0.083$ (i.e., less than .085 ), $\mathrm{SRMR}=0.039$ (i.e., less than .08). One restricted model was tested (Model 2 nested within Model 1) to see whether a simpler conceptualization of anxiety sensitivity might provide a better fit to the data than did Model 1 (the full model). The models that were compared are listed in Table 3, along with their fit statistics.

The model-fitting process suggested that Model 2 (Model 2) best fit the data: $\chi^{2}(0)=0$ (i.e., under 3), $\mathrm{CFI}=1.000$ (i.e., greater than .95 ), $\mathrm{TLI}=1.000$ (i.e., greater than .95), RMSEA $=0.000$ (i.e., less than .08), $\mathrm{SRMR}=0.000$ (i.e., less than .08). The model with the lowest AIC is preferred. Model 2 had the lowest AIC. On 
this basis, Model 2 was selected as the best model of all the models and based further analyses on that model. In addition, more focused fit tests (examination of modification indices, offending estimates, standardized residuals and evaluations of theoretical coherence) all suggested adequate model fit. Model 2 is called the trimmed full model (Figure 1).

The path diagram that shows the fully-unstandardized parameter estimates for Model 2 appears in Figure 1. The $\mathrm{R}^{2}$ values were 0.391 for RCMAS. Model 2 (the trimmed full model) as a whole accounted for $39 \%$ of the variance in RCMAS.

\section{Maternal Acculturation Effects}

A main objective of this study was to evaluate the respective effects of parent acculturation and country of origin on youth anxiety.

Dominant Society Immersion and Ethnic Society Immersion. Maternal acculturation was measured with the two subscales of the SMAS (Stephenson, 2000), the ESI and the DSI. The ESI showed a statistically significant path coefficient of $0.068(\mathrm{p}<.05)$. That is, for every one unit increase in ESI, youth anxiety increased 0.068 units. There was not a statistically significant path coefficient from the DSI to youth anxiety. Mother Country of Origin

Two dummy variables were constructed to indicate non-Cuban Hispanic $(\mathrm{n}=155)$ and Cuban Hispanic $(\mathrm{B}=449)$ in the study's sample. Using youth self ratings of anxiety (i.e., RCMAS), a multiple group model with regression paths constrained to be equal across country of origin did not provide a good fit: $\chi^{2}(3748.460)=33$ (i.e., greater than 3), $\mathrm{CFI}=0.015$ (i.e., less than .95 ), $\mathrm{TLI}=-0.432$ (i.e., less than .95 ), $\mathrm{RMSEA}=0.387$ (i.e., greater than .08 ), $\mathrm{SRMR}=0.105$ (i.e., greater than .08 ). Relaxing 
the path equality constraint did not significantly improve the model fit, $\chi^{2}(2761)=30$ (i.e., greater than 3), $\mathrm{CFI}=0.020$ (i.e., less than .95), $\mathrm{TLI}=1.000$ (i.e., greater than $.95), \mathrm{RMSEA}=0 . .286$ (i.e., greater than .08$), \mathrm{SRMR}=0.205$ (i.e., greater than .08 ), $\mathrm{p}$ $<.01$.

Anxiety Sensitivity

Another main objective of the present study was to measure AS and its relation to anxiety. Also, the variables of youth age and youth sex were included as potential moderators of the relation between AS and anxiety symptoms. Other interaction effects between anxiety sensitivity and youth age were also tested. Youth Age*Anxiety Sensitivity. Given that youth age is a continuous variable, its moderating effect on the relation between AS and youth anxiety symptoms was evaluated using the product term approach (Jaccard, Turrisi \& Wan, 1990 Jaccard \& Wan, 1996). There was not a significant interaction between age and AS ( $>>.05)$. These results suggest a lack of significant interaction effects of youth age on the moderated relation between AS and youth anxiety symptoms (i.e., RCMAS). Youth Sex* Anxiety Sensitivity. To evaluate a moderating effect of youth sex on the relation between AS and youth anxiety symptoms, two dummy variables were constructed to indicate males $(n=294)$ and females $(n=310)$ in the study's sample. Using youth ratings of anxiety (i.e., RCMAS), a multiple group model with regression paths constrained to be equal across youth sex did not provide a good fit: $\chi^{2}(3742.192)$ $=33$ (i.e., greater than 3), $\mathrm{CFI}=0.016$ (i.e., less than .95), $\mathrm{TLI}=-0.432$ (i.e., less than $.95), \mathrm{RMSEA}=0.387$ (i.e., greater than .08 ), $\mathrm{SRMR}=0.153$ (i.e., greater than .08 ). Relaxing the path equality constraint significantly improved the model fit, $\chi^{2}(24)=3$ 
(i.e., under 3), $\mathrm{CFI}=0.98$ (i.e., greater than .95 ), $\mathrm{TLI}=0.96$ (i.e., greater than .95 ), RMSEA $=0.04$ (i.e., less than .08 ), $S R M R=0.05$ (i.e., less than .08 ). The residual for females was .614 . That is, being female predicted youth anxiety symptoms $(B=.614$; $\mathrm{SE}=.025, \mathrm{p}<.05)$ The residual for males was .535 . That is being male also predicted youth anxiety symptoms $(\mathrm{B}=.415, \mathrm{SE}=.011, \mathrm{p}<.05)$.

RCMAS Lie Scale

Lastly, the examination of the contribution of RCAMS' Lie Scale to youth anxiety scores was an objective of the study. The RCMAS Lie Scale showed a statistically significant path coefficient of $0.427(\mathrm{p}<.05)$. That is, for every one unit increase in the RCMAS Lie Scale, youth anxiety increased 0.427 units. 


\section{Chapter V.}

\section{Discussion}

The present study aimed to evaluate whether: (1) a CFA would yield a twofactor structure of the SMAS (Stephenson, 2000); (2) maternal acculturation as measured by the SMAS is related to youth anxiety symptoms; (3) mother country of origin (i.e. Cuban or another Latin country) moderates the relationship between youth anxiety sensitivity and youth anxiety symptoms; (4) youth age moderates the relationship between youth anxiety sensitivity and youth anxiety symptoms; (5) youth sex moderates the relationship between youth anxiety sensitivity and youth anxiety symptoms; (6) the RCMAS Lie Scale is related to Hispanic youths' reporting of anxiety symptoms.

\section{Summary of Dissertation Findings}

With respect to the factor structure of the SMAS (Stephenson, 2000) in the current sample, a CFA revealed a two-factor structure that supported the Ethnic Society Immersion (ESI) and Domestic Society Immersion (DSI) subscales of the SMAS. Two items $(12,25)$ double loaded on both scales and one item (4) did not load on either factor. With respect to the relation between maternal acculturation and youth anxiety symptoms, the results revealed that ESI was related to youth anxiety symptoms. That is, as the mother's score on ESI increased, or the more immersed they were in their ethnic society, the more anxiety symptoms youth reported.

With respect to whether mother's country of origin (i.e., Cuba or another Latin country) moderated the relation between AS and youth anxiety symptoms, the results 
revealed no significant difference between youths' whose mothers' country of origin was a non-Cuban Latin country and youth whose mothers country of origin was Cuba.

With respect to whether youth age impacted the relation between AS and youth anxiety symptoms, the results revealed no statistically significant difference as youth age increased.

With respect to whether youth sex moderated the relation between AS and youth anxiety symptoms, the results revealed that being female was more likely to moderate the relationship between AS and anxiety than being male.

With respect to the relation between the RCMAS Lie Scale and youth anxiety symptoms, the results revealed that the RCMAS Lie Scale was related to youth anxiety symptoms. That is, the higher a youth scored on the RCAMS Lie Scale, the more anxiety symptoms they reported.

\section{Contribution of the Present Study and Implications}

The present study contributes to the current literature on theoretical, empirical, and clinical levels. The main contributions on each of these levels are summarized below including potential implications.

Theoretical Implications. In the present study, acculturation was defined as degree of immersion in dominant (DSI subscale) and ethnic societies (ESI subscale). Degree of immersion was measured as superficial and intermediate behaviors at the individual level in the domains of language, interaction, food, and media. Operationalizing acculturation using two subscales (DSI and ESI) is conducive to its application across ethnic groups because it does not presume to measure cultural change or the acquisition of new beliefs and values, or to capture the meaning of 
change among acculturating individuals. By confirming the two-factor structure of the SMAS (Stephenson, 2000), this study furthered the evidence that acculturation can be measured in different Hispanic groups via the SMAS.

The study also extends past research in that it is the first study to examine if a link exists between mother's level of acculturation and youth anxiety. Although studies have been conducted using proxy variables such as parent primary language choice (i.e., English, Spanish), parent ethnicity and parent nativity, there have not been any studies examining the impact of parent acculturation on youth anxiety using a continuous acculturation measure in Hispanic youth. Although the more mothers were immersed in ethnic society was related to their youth reporting more anxiety symptoms, this result was not found for mothers' immersion in dominant society. The differences found related to ethnic society immersion are theoretically relevant as it suggests that immersion in ones' own ethnic society does not have protective effect in this particular sample. The 'immigrant paradox' that has been observed in MexicanAmerican samples does not hold true for this sample of Hispanics. Differences could be the result of a variety of differences between Hispanic subgroups.

Conversely, results of the current study did not support the hypothesis that the mother's country of origin (Cuban descent or other Hispanic country) would change the relationship between AS and anxiety symptoms. In fact, there was not a relationship at all between mother country of origin and youth anxiety symptoms. Theoretically, the lack of relationship between mother country or origin and youth anxiety symtpoms could imply that membership in a specific Hispanic subgroup is not related to anxiety. 
The relation between the RCMAS Lie Scale and the youths' reporting of anxiety symptoms in the current sample could indicate a difference in cultural norms for Hispanic families. For example, the child's endorsement of items like "I like everyone I know" or "My child likes everyone he/she knows" could reflect collectivistic qualities of Hispanic culture as opposed to being an indicator of defensiveness on the part of the participants. Numerous cultural theorists propose that Hispanic culture places emphasis on emotional restraint. Emotional restraint within Hispanic society is linked to a collectivistic ideal of putting the whole above oneself. The construct of simpatia (good nature or pleasant attitude) refers patterns of social interaction and verbal communication that emphasize a pleasant attitude aimed to promote agreement and reduce or avoid conflict (Mezzich, Ruiz, \& Munoz, 1999). It encapsulates a common desire to have warm and pleasurable social relationships by promoting smooth communication, conformity, and cooperation (Marin \& Marin, 1991; Santiago-Rivera, 2003). Therefore, the relation between the RCMAS Lie scale and youths' reporting of anxiety symptoms could be due to youths' conceptions of simpatia and the collectivistic culture in Hispanic groups.

The present study provides support for the importance of considering AS, ethnicity and acculturation in anxiety psychopathology in Hispanic youth. The relation between AS and anxiety symptoms indicates that Hispanic youth may be more aware of anxiety-related somatic symptoms (AS). In turn, they report more anxiety symptoms. Therefore, the mechanism driving the higher reporting of anxiety symptoms in Hispanic youth samples may be related to a heightened awareness about anxiety symptoms in Hispanic youth instead of more youth-reported anxiety 
symptoms. In regard to the impact of acculturation on youth anxiety, maternal ethnic society immersion increased youth-reported anxiety symptoms. Taken together, these findings indicate that there is a possible relation between AS and acculturation in Hispanic youth.

Empirical Implications. The present study is, to some extent, consistent with previous studies in some of the findings. With regard to the two-factor model of the SMAS, the current study is consistent with past studies (Stephenson, 2000) in supporting a two-factor model for the SMAS. The current study also adds to empirical knowledge about the validity of the SMAS in Hispanic minority samples. The current sample is primarily composed of Cuban-American and Central American parents while the samples in factor analytic studies of the SMAS have been composed of Mexican-Americans.

The current study also replicated Varela (2007) in that Hispanic females reported more anxiety symptoms than Hispanic males. Also, further analysis indicated that being female increased the relationship between AS and youth anxiety more than being male. Taken together, these findings add to empirical knowledge that sex differences do exist in Hispanic youth with regard to anxiety sensitivity and anxiety.

Clinical Implications. This study provides further support to the need for interventions that can be tailored towards Hispanic youth with anxiety disorders. It is predicted that nearly one-third of those under 19 years of age will be Hispanic by 2050 (Spencer \& Hollmann, 1998). Individuals of Mexican origin comprise the largest proportion of Latinos (almost two-thirds), with the remaining third distributed primarily among persons of Puerto Rican, Cuban, and Central American origin. It is 
noteworthy that nearly two-thirds of Hispanics (64 \%) were born in the United States (U.S. Census Bureau, 2000c).

Given the high proportion of Hispanics now being born in the United States, interventions that are tailored towards the growing needs of US-born Hispanic youth are increasingly necessary. The current study brought up two important results in terms of how acculturation and Hispanic group membership can impact youth anxiety symptoms: Hispanic youth whose parents report more immersion in their ethnic society report more anxiety symptoms and Hispanic youth who report higher on the RCMAS Lie Scale report more anxiety symptoms. Clinically, addressing issues beyond anxiety reduction to a more broad intervention to help youth feel more acculturated could help in further reducing anxiety symptoms in Hispanic youth.

Additionally, addressing culturally-specific constructs of Hispanics and Hispanic subgroups could further enhance investigations into AS, anxiety and clinical interventions. Organista (2000) stated that despite the diversity of Hispanic groups in the U.S., they share common elements, including their family roots in Latin American countries, their Spanish language, and their cultural traditions resulting from the blend of Spanish colonists and the indigenous peoples of the Americas. In recent years, the literature suggests (Comas-Diaz, 1997; Falicov, 1998; Flores, 2000; Marin \& Marin, 1991; Santiago-Rivera, 2003; Santiago-Rivera, Arredondo, \& Gallardo-Cooper, 2002) that psychologists need to be aware of the potential implications of certain cultural characteristics found within the Hispanic community in order to guide their interpersonal behaviors during the delivery of treatment services. 
Specific to the present study, culturally-specific constructs could be the underlying mechanism in the relationship between AS and anxiety. Perhaps, the concept of deshago (getting things off one's chest) mediates or moderates the relationship between AS and anxiety. Many Hispanic/Latino clients believe that the purpose of treatment is to have the chance for desahogo (Martinez-Guarnaccia, 2007). Adherenace to deshago brings up the possibility that Hispanic youth may be not only more aware of their anxious symptoms, but may feel more compelled to share their anxious symptoms and feelings when compared to other ethnic groups.

\section{Limitations and Future Research Directions}

It is important to note some limitations of the study. One notable limitation is that the comparison of mothers' country of origin was limited to those who were of Cuban origin compared to other Hispanic groups. Perhaps, the lack of differences between the two groups resulted from aggregation all of the "other" Hispanic countries into one group. A sample that is more representative of US Hispanics (i.e., Mexican, Cuban, Puerto Rican and Central American) could yield different results. Although the location of the data collection limited the number of mothers of Mexican and Puerto Rican origin, further collection of data from mothers of Central American origin is a possible extension of the current study.

Second, acculturation was limited to measurement of maternal acculturation, which limits the results as the anxiety measured was youth anxiety. A youth measure of acculturation could add to the findings in that comparisons could be made to the effects of maternal acculturation and youth acculturation on AS and anxiety symptoms. 
Third, the measures used in this study were translated carefully, however, they were not developed specifically for use with the Hispanic population. Future research with Hispanic youth should focus on measuring culture specific versus general meanings of AS and anxiety.

Fourth, the youth that composed the sample in the study were referred to an anxiety clinic for clinical issues relating to worries and fears. Given that anxious symptoms and anxiety are universal to all people on some level, future studies could investigate community or school samples of Hispanic youth for relationships between anxiety sensitivity, maternal acculturation, country of origin and youth anxiety. Using a community or school sample could improve upon and expand the study in two ways. First, it could capture those youth who do not seek treatment for clinical anxiety due to barriers such as transportation or financial resources. Second, using a school or community sample would not only expand the sample size but also increase the number of Hispanic subgroups, as referenced above.

Lastly, the current study is the cross-sectional nature of the data collected. More time points where the same data is collected could ascertain if changes in maternal acculturation (either an increase in dominant society immersion or a decrease in ethnic society immersion) affect youth reported anxiety symptoms. 


\section{LIST OF REFERENCES}

Allen, M. L., Elliott, M. N., Fugligni, A. J., Morales, L. S., Hambarsoo- mian, K., \& Schuster, M. A. (2008). The relationship between Spanish language use and substance use behaviors among Latino youth: A social network approach. Journal of Adolescent Health, 43, 372-379. doi:10.1016/j.jadohealth.2008.02.016

Anderson, Emily R., \& Mayes, Linda C. (2010). Race/ethnicity and internalizing disorders in youth: A review. Clinical Psychology Review (30). 338-348. Doi: 10.1016/j.cpr.2009.12.008

Bates, L., Acevedo-Garcia, D., Alegria, M., \& Krieger, N. (2008) Immigration and generational trends in body mass index and obesity in the United States: Results of the National Latino and Asian-American Survey (NLAAS), 2002-2003. American Journal of Public Health. Doi: 10.2105/AJPH.2006.102814

Beidel, D. C. (1991). Social phobia and overanxious disorder in school age children. Journal of the American Academy of Child and Adolescent Psychiatry, $30,545-552$.

Chorpita, B. F., Albano, A. M., \& Barlow, D. H. (1996). Child anxiety sensitivity: Considerations for children with anxiety disorder. Journal of Clinical Child Psychology, 25, 77-82.

Comas-Diaz, L. (1997). Mental health needs of Latinos with professional status. In J.G. Garcia \& M. C. Zea (Eds.), Psychological interventions and research with Latino populations (pp. 142-165). Needham Heights, MA: Allyn \& Bacon.

Costello, J. E., Egger, H. L., Copeland, W., Erkanli, A., \& Angold, A. (2009). The developmental epidemiology of anxiety disorders: Phenomenology, prevalence, and comorbidity. In W. K. Silverman, \& A. Field (Eds.), Anxiety Disorders in Children and Adolescents: Research, Assessment and Intervention. Cambridge University Press.

Crespo CJ, Smit E, Carter-Pokras O, Andersen R. (2001)Acculturation and leisuretime physical inactivity in Mexican American adults: results from NHANES III, 1988-1994. American Journal of Public Health, (91), 1254 -12457. doi: 10.2105/AJPH.91.8.1254

Cuéllar, I. (2000). Acculturation and mental health: Ecological transactional relations of adjustment. In I. Cuéllar and F. A. Paniagua (Eds.) Handbook of multicultural mental health (pp 45-63). San Diego, CA: Academic Press. 
Cuéllar, I., \& Roberts, R. E. (1997). Relations of depression, acculturation, and socioeconomic status in a Latino sample. Hispanic Journal of Behavioral Sciences, 19, 230-238.

Falicov, C. F. (1998). Latino families in therapy: A guide to multicultural practice. New York: Guilford. competency in health care. The Journal of Pediatrics, 136, 14-23.

Gibson, M. A. (2001). Immigrant adaptation and patterns of acculturation. Human Development, 44, 19-23. doi:10.1159/000057037.

Ginsburg, G.S., Riddle, M.A. \& Davies, M. (2006) Journal of the American Academy of Child and Adolescent Psychiatry, 44(10), 1179-1187.

Glover, S.H., Pulmareiga, A.J., Holzer, C.E., Wise, B.K. \& Rodriguez, M. (1999). Anxiety symptomatology in Mexican-American adolescents. Journal of Child and Family Studies, 8(1), 47-57. doi: 10.1023/A:1022994510944.

Jaccard, J. (1998). Interaction effects in factorial analysis of variance. Newbury Park, CA: Sage.

Jaccard, J., \& Turrisi, R. (2003). Interaction effects in multiple regression (2nd ed.). Thousand Oaks, CA: Sage.

Jaccard, J., Turrisi, R., \& Wan, C. (1990) Interaction effects in multiple regression. Newbury Park: Sage.

Jaccard, J., \& Wan, C. (1996). LISREL analyses of interaction effects in multiple regression. Newbury Park, CA: Sage.

King, G., Honaker, J., Joseph, A., \& Scheve, K. (2001). Analyzing incomplete political science data: An alternative algorithm for multiple imputation. American Political Science Review, 95, 49-69.

Last, C. G., Hersen, M., Kazdin, A. E., Orvaschel, H., \& Perrin, S. (1991). Anxiety disorders in children and their families. Archives of GeneralPsychiatry, 48, 928934.

Lilienfeld, S. O., Jacob, R. G., \& Turner, S. M. (1989). Comment on Holloway and McNally's (1987) "Effects of anxiety sensitivity on the response to hyperventilation". Journal of Abnormal Psychology, 98, 100-102.

Magaña, G. S., De la Rocha, O., Amsel, H. A., Fernandez, M. I., \& Rulnick, S. (1996). Revisiting the dimensions of acculturation: Cultural theory and psychometric practice. Hispanic Journal of Behavioral Sciences, 18 (4), 444-468. 
March, J. S., Parker, J. D. A., Sullivan, K., \& Stallings, P. (1997). The Multidimensional Anxiety Scale for Children: Factor structure, reliability and validity. Journal of the American Academy of Child \& Adolescent Psychiatry,(36), 554-565. Doi: 10.1097/00004583-199704000-00019

Marian, E., \& McNally, R. J. (1996). Anxiety sensitivity, suffocation, fear, trait anxiety, and breathholding. Behaviour Research and Therapy, 34, 603-607.

Marin, G., \& Marin, B. V. (1991). Research with Hispanic populations. Newbury Park, CA: Sage.

McNally, R. J. (1996). Anxiety sensitivity is distinguishable from trait anxiety. In R. M. Rapee (Ed.), Current Controversies in the Anxiety Disorders (pp. 245-248). New York: Guilford Press.

McNally, R. J. (1989). Is anxiety sensitivity distinguishable from trait anxiety: a reply to Lilienfeld, Jacob and Turner? Journal of Abnormal Psychology, 98, 193-94.

McNally, R. J. (1999). Theoretical approaches to the fear of anxiety. In S. Taylor (Ed.), Anxiety Sensitivity: Theory, Research and Treatment (pp. 3-16). London: Lawrence Erlbaum Associates.

Muris, P., Schmidt, H., Merckelbach, H., \& Schouten, E. (2001). Anxiety sensitivity in adolescents: factor structure and relationships to trait anxiety and symptoms of anxiety disorders and depression. Behaviour Research and Therapy, 39, 89-100.

Muthén, L.K. and Muthén, B.O. (1998-2007). Mplus User's Guide. Fifth Edition. Los Angeles, CA: Muthén \& Muthén.

Noel, V.A. \& and Francis, S.E. (2011). A meta-analytic review of the role of child anxiety sensitivity in child anxiety. Journal of Abnormal Child Psychology, 39(5), 721-733. doi: http://dx.doi.org/10.1007/s10802-011-9489-3

Olatjuni, B. O., \& Wolitzky-Taylor, K. B. (2009). Anxiety sensitivity and the anxiety disorders: a meta-analytic review and synthesis. Psychological Bulletin, 135, 974- 999.

Pina, A., \& Silverman, W.K. (2004). Phenomenology, somatic symptoms, and distress in hispanic/latino and european american youth with anxiety disorders. Journal of Clinical Child and Adolescent Psychology, 33, 227-236. doi: 10.1207/s15374424jccp3302_3

Pina, A., Silverman, W.K. \& Weems, C. (2001). An analysis of the RCMAS lie scale in a clinic sample of anxious children. Journal of Anxiety Disorders, 15, 443-447 doi: http://dx.doi.org/10.1016/S0887-6185(01)00075-5 
Reiss, S. (1991). Expectancy model of fear, anxiety, and panic. Clinical Psychology Review, 11, 141-153. doi: 10.1016/0272-7358(91)90092-9

Reiss, S., Peterson, R. A., Gursky, D. M., \& McNally, R. J. (1986). Anxiety sensitivity, anxiety frequency and the predictions of fearfulness. Behaviour Research and Therapy, 24, 1-8. Doi: 10.1016/0005-7967(86)90143-9.

Reiss, S., Silverman, W. K., \& Weems, C. F. (2001). Anxiety sensitivity. In M. W. Vasey, \& M. R. Dadds (Eds.), The Developmental Psychopathology of Anxiety (pp. 92-111). New York: Oxford University Press.

Reynolds, C. R., \& Richmond, B. O. (1978). What I think and feel: A revised measure of children's manifest anxiety. Journal of Abnormal Child Psychology, 6, 271-280.

Roberts, R.E., Roberts, C.R. \& Xing, Y. Prevalence of youth-reported DSM-IV psychiatric disorders among african, european, and mexican american adolescents. Journal of the Academy of Child and Adolescent Psychiatry, 45(11), 1329-1337. doi: 10.1097/01.chi.0000235076.25038.81

Santiago-Rivera, Azara. (2003). Latinos values and family transitions: Practical considerations for counseling. Counseling and Human Development, 35, 1-12.

Santiago-Rivera, A. L., Arredondo, P., \& Gallardo-Cooper, M. (2002). Counseling Latinos and la familia: A practical guide. Thousand Oaks, CA: Sage.

Silverman, W. K., \& Albano, A. M. (1996). Anxiety Disorders Interview Schedule for Children-IV (Child and Parent Versions). San Antonio, TX: Psychological Corporation.

Spielberger, C. D. (1973). Manual for the State-Trait Anxiety Inventory for Children. Palo Alto, CA: Consulting Psychologists Press.

Taylor, S., \& Fedoroff, I. C. (1999). The expectancy theory of fear, anxiety and panic: a conceptual and empirical analysis. In S. Taylor (Ed.), Anxiety Sensitivity: Theory, Research and Treatment of the Fear of Anxiety (pp. 17-34). London: Lawrence Erlbaum Associates.

Umana-Taylor, A.J. \& Alfaro, Edna, C. (2009). Acculturative stress and adaptation. In Handbook of U.S. Latino psychology: Developmental and community-based perspectives, 135-152. Thousand Oaks, CA: Sage Publications.

Varela, R. E., Weems, C. F., Berman, S. L., Hensley, L., \& Rodriguez de Bernal, M. C. (2007). Internalizing symptoms in Latinos: The role of anxiety sensitivity. Journal of Youth and Adolescence, 36, 429-440. doi:10.1007/s10964-007-91684. 
Varela, R. E., Sanchez-Sosa, J. J., Biggs, B. K., \& Luis, T. M. (2008). Anxiety symptoms and fears in Hispanic and European American children: Cross-cultural measurement equivalence. Journal of Psychopathology and Behavioral Assessment, 30, 132-145. doi:10.1007/s10862-007-9056-y.

Varela, R.E., Hensley-Maloney, L. (2009). The influence of culture on anxiety in latino youth: A review. Clinical Child and Family Psychology Review, 12, $217-$ 233. doi: 10.1007/s10567-009-0044-5

Warner, L.A., Valdez, A., Vega, W.A., de la Rosa, M., Turner, R.J.\& Canino, G. (2006). Hispanic drug abuse in an evolving cultural context: An agenda for research. Drug and Alcohoal Dependence, 84, S8-S16.

Weems, C. F., Hammond-Laurence, K., Silverman, W. K., \& Ginsburg, G. (1998). Testing the utility of the anxiety sensitivity construct in children and adolescents referred for anxiety disorders. Journal of Clinical Child Psychology, 24, 69-77.

Weems, C.F., Hayward, C., Killen, J., Taylor, C.B. (2002). A longitudinal investigation of anxiety sensitivity in adolescence. Journal of Abnormal Psychology, 111 (3), 471-477. doi: http://dx.doi.org/10.1037/0021843X.111.3.471

Weems, C.F., Taylor, L.K., Marks, A.B., Varela, R.E. (2010). Anxiety sensitivity in childhood and adolescence: Parent reports and factors that influence associations with child reports. Cognitive therapy and research, 34(4), 303-315doi:

10.1007/s10608-008-9222-x 
Table 1

Items Contributing to Factors and CFA Loading Items

Factor 1 Factor 2

1. I understand English, but I'm not fluent in English.

$-.472$

2. I am informed about current affairs in the United States.

.432

3. I speak my native language with my friends and acquaintances from my country of origin.

4. I have never learned to speak the language of my native country.

5. I feel totally comfortable with (Anglo) American people. $\quad .726$

6. I eat traditional foods from my native country.

7. I have many (Anglo) American acquaintances.

.770

8. I feel comfortable speaking my native language.

9. I am informed about current affairs in my native country.

10. I know how to read and write in my native language.

11. I feel at home in the United States.

12. I attend social functions with people from my native

country.

13. I feel accepted by (Anglo) Americans.

14. I speak my native language at home.

15. I regularly read magazines of my ethnic group.

16. I know how to speak my native language.

17. I know how to prepare (Anglo) American foods.

18. I am familiar with the history of my native country.

19. I regularly read an American newspaper.

20. I like to listen to music of my ethnic group.

21. I like to speak my native language.

22. I feel comfortable speaking English.

23. I speak English at home.

24. I speak my native language with my spouse or partner.

25 . When I pray, I use my native language.

26. I attend social functions with (Anglo) American people.

27. I think in my native language.

28 . I stay in close contact with family members and relatives

in my native country.

29. I am familiar with important people in American history. $\quad .745$

30. I think in English.

31. I speak English with my spouse or partner.

32. I like to eat American foods. 
Table 2

Defined Models

Model Description

SEM Model

Model 1

Full Model

Model 2

One path (youth depression-> anxiety sensitivity) was fixed to zero.

Note. Model 2 is nested within Model 1. 
Table 3

Fit indices for Each of the Models

\begin{tabular}{lllllll}
\hline Model & $\chi^{2}$ & df & CFI & TLI & RMSEA SRM & AIC
\end{tabular}

BIC

SEM Model

Model 67.869

9989.105

Model 20

5176.902

\begin{tabular}{|c|c|c|c|c|c|}
\hline 11 & 0.975 & 0.944 & 0.083 & 0.039 & 9905.920 \\
\hline 0 & 1.000 & 1.000 & 0.000 & 0.000 & 5107.581 \\
\hline
\end{tabular}

Note. Model 2 is nested within Model $1 . \mathrm{CFI}=$ comparative fit index; TLI $=$ NNFI $=$ nonnormed fit index; RMSEA = root-mean-square error of approximation; SRMR = standardized root mean-square residual; $\mathrm{AIC}=$ akaike information criterion; $\mathrm{BIC}=$ bayesian information criterion; Model $1=$ full model (all free); Model $2=$ One path (youth depression-> anxiety sensitivity) was fixed to zero. 
Figure 1

Final Model with Significant Paths

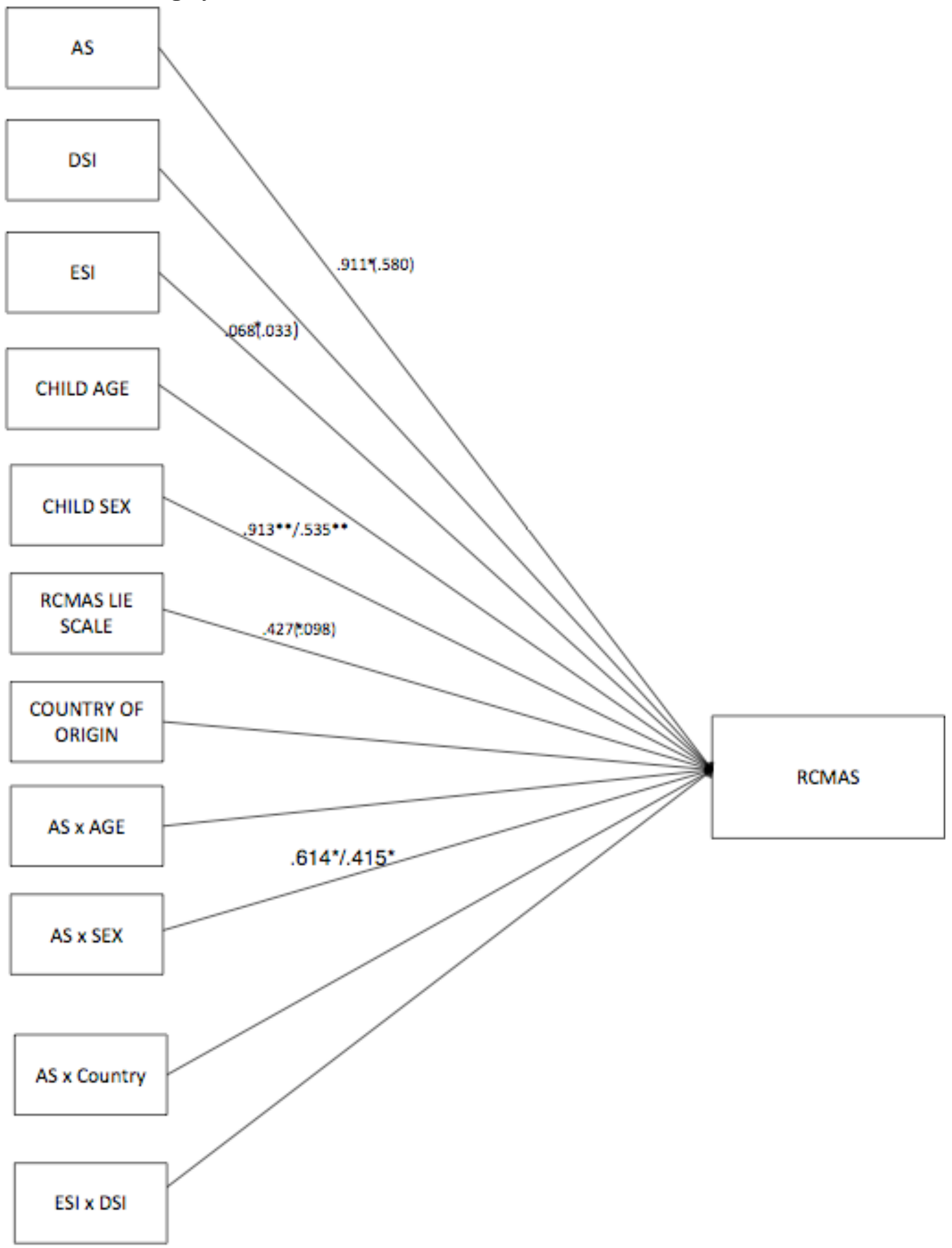


VITA

\section{MARIA PIENKOWSKI}

B.S., Child Development

Vanderbilt University

Nashville, TN

2006

M.Ed., Applied Child Studies

Vanderbilt University

Nashville, TN

2012

M.S. Developmental Psychology

Miami, FL

2012 to present

Doctoral Candidate

Florida International University

Miami, FL

\section{PUBLICATIONS AND PRESENTATIONS}

Pienkowski, M., Marin, C., Rey, Y. \& Silverman, W. (2011, August). Locus of control and anxiety in hispanic youth. Poster session presented at the American Psychological Association Conference, Washington D.C.

Pienkowski, M., Gray, C., Hernandez, I., Sangiovanni, P., Alonso, A., Carter, R., \& Silverman, W. (2008, March). Test anxiety in elementary school children: re-examining an "old" but useful construct. Poster session presented at the 28th Annual Conference of the Anxiety Disorders Association of America, Savannah, GA.

Perez, N. A., Brown, S., Compton, C., Pienkowski, M. \& Nash, M. R. (2005).

Suggestibility, Hypnotizability and Personality. Paper Presented at the Annual Conference of the Society for Clinical and Experimental Hypnosis, Charleston, NC. 
